\title{
On computational Aspects of Bismuth Tri-Iodide
}

Abaid ur Rehman Virk ${ }^{2}$, Waqas Nazeer ${ }^{3, *}$ and Shin Min Kang ${ }^{4}$

1. Department of Mathematics, University of Management and Technology, Lahore Pakistan; abaid.math@gmail.com

2. Department of Mathematics, Division of Science and Technology, University of Education Lahore Pakistan

3. Department of Mathematics and RINS, Gyeongsang National University, Jinju 52828, Korea, smkang@gnu.ac.kr

Corresponding Author: nazeer.waqas@ue.edu.pk ; +92-321-4707379

\begin{abstract}
The topological index is a numerical quantity based on the characteristics of various invariants or molecular graph. For ease of discussion, these indices are classified according to their logical derivation from topological invariants rather than their temporal development. Degree based topological indices depends upon the degree of vertices. This paper computes Zagreb polynomials and redefined first, second and third Zagrebindices of Bismuth Tri-Iodide chains and sheets.
\end{abstract}

Keywords: topological index, Bismuth Tri-Iodide, Molecular graph, Zagreb index, Randic index, Zagreb polynomial.

\section{Introduction}

$\mathrm{BiI}_{3}$ is an inorganic compound which is the result of the reaction of iodine and bismuth, which inspired the enthusiasm for subjective inorganic investigations [1]. $\mathrm{BiI}_{3}$ is an excellent inorganic compound and is very useful in qualitative inorganic analysis [2].

It has been proven that $B i$-doped glass optical strands are one of the most promising dynamic laser media. Different kinds of Bi-doped fiber strands have been created and have been used to construct Bi-doped fiber lasers and optical loudspeakers [3].

Layered $\mathrm{BiI}_{3}$ gemstones are considered to be a three-layered stack structure in which a plane of bismuth atoms is sandwiched between iodide particle planes to form a continuous $I-B i-I$ plane [4].

The periodic superposition of the three layers forms diamond-shaped $\mathrm{BiI}_{3}$ crystals with $R-3$ symmetry [5,6]. A progressive stack of $I-B i-I$ layers forms a hexagonal structure with symmetry [7]. A jewel of $\mathrm{BiI}_{3}$ has been integrated in [8]. 


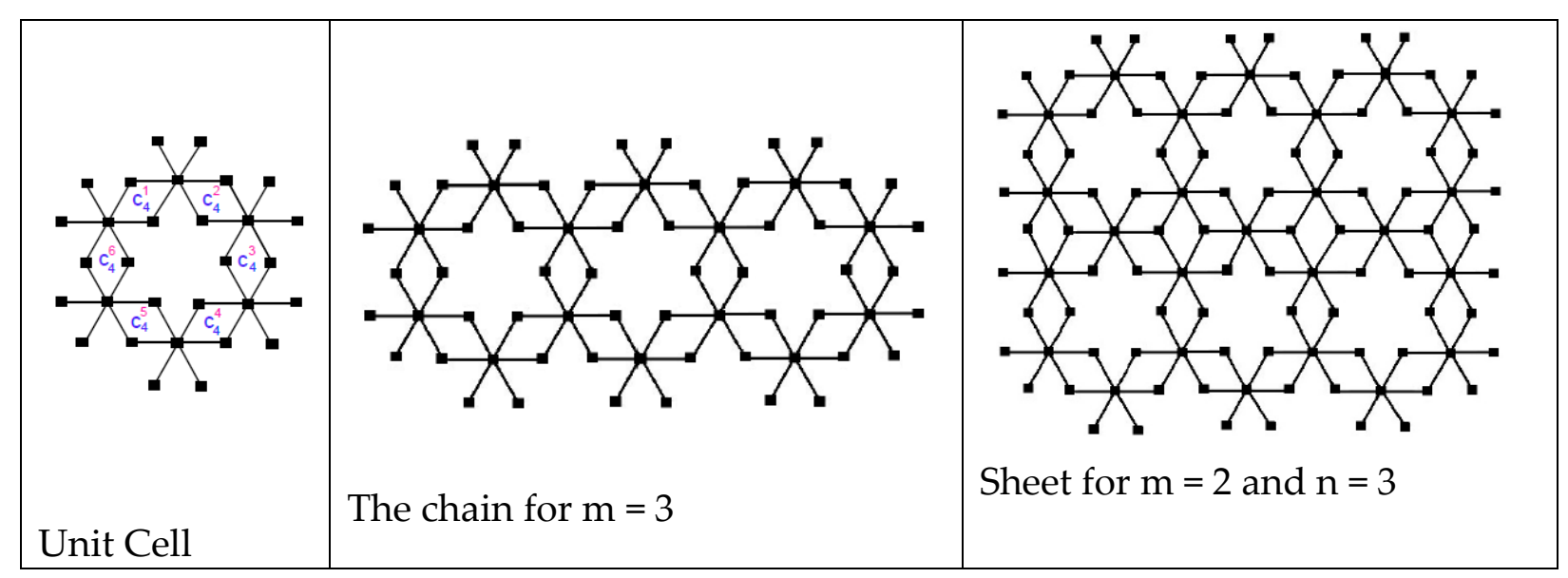

Figure 1 bismuth tri-iodide

In the unit cell (figure 1), Main cycles are $C_{4}^{1}, C_{4}^{2}$ central cycles are $C_{4}^{3}, C_{4}^{6}$ and Base cycles are $C_{4}^{4}, C_{4}^{5}$

Given its enormous application in the science of impurity-free and connection, graph theory is a multidimensional topic. It is feasible to display and plan crystal structures, complex systems, and synthesis charts. There are many compounds that are organic and inorganic compounds that can be used in commercial, industrial and laboratory environments as well as in everyday life. There is a relationship between the synthesis mixture and its atomic structure. Graph theory is an effective field of arithmetic and has a huge range of applications in many scientific fields such as chemistry, software engineering, electrical and electronics. Chemical graph theory is other branches of science in which graphs are used to show the mixture graphically using proficient instruments.

The physical structure of a strong material usually depends on the action of atoms, particles, or atoms that make up a strong bond between them. The crystal structure, also known as crystal material or crystal strength, is made of a unit cell, which is organized in $3 \mathrm{D}$ on the grid. The scheme of atomic or crystalline materials is crucial for determining the behavior and properties of materials such as metals, composites, and art materials. Cells are the smallest auxiliary units that can clarify the gem structure (unit cell). The redundancy of the cell creates the entire structure.

Mathematical chemistry provides tools such as polynomials and functions to capture information hidden in the symmetry of molecular graphs and thus predict properties of compounds without using quantum mechanics. A topological index isa numerical parameter of a graph and depicts its topology. It describes the structure of molecules numerically and are used in the development of qualitative structure activity relationships (QSARs). Most commonly known invariants of such kinds are degree- 
based topological indices. These are actually the numerical values that correlate the structure with various physical properties, chemical reactivity and biological activities [9-13]. It is an established fact that many properties such as heat of formation, boiling point, strain energy, rigidity and fracture toughness of a molecule are strongly connected to its graphical structure.

Hosoya polynomial, (Wiener polynomial), [14] plays a pivotal role in distance-based topological indices. A long list of distance-based indices can be easily evaluated from Hosoya polynomial. A similar breakthrough was obtained recently by Klavzar et. al. [15], in the context of degree-based indices. Authors in [15] introduced M-polynomial in, 2015, to play a role, parallel to Hosoya polynomial to determine closed form of many degree-based topological indices [16-20]. The real power of M-polynomial is its comprehensive nature containing healthy information about degree-based graph invariants. These invariants are calculated on the basis of symmetries present in the $2 \mathrm{~d}-$ molecular lattices and collectively determine some properties of the material under observation.

In this article, we compute general form of Zagreb polynomials for Bismuth Tri-Iodide chains and Bismuth Tri-Iodide sheets. Then we derive closed forms of redefined first, second and third Zagreb indices for Bismuth Tri-Iodide.

\section{Basic definitions and Literature Review}

Throughout this article, we assume $\mathrm{G}$ to be a connected graph, $V(G)$ and $E(G)$ are the vertex set and the edge set respectively and $d_{v}$ denotes the degree of a vertex $v$.

The first and second Zagreb indices are one of the oldest and most well-known topological indices defined by Gutman in 1972 and are given different names in the literature, such as the Zagreb group index, Sag. Loeb group parameters and the most common Zagreb index. The Zagreb index is one of the first indices introduced and has been used to study molecular complexity, chirality, ZE isomers and heterogeneous systems. The Zagreb index shows the potential applicability of deriving multiple linear regression models.

The first and the second Zagreb indices [21] are defined as

$$
M_{1}(G)=\sum_{u v \in E(G)}\left(d_{u}+d_{v}\right)
$$

and 
$M_{2}(G)=\sum_{u v \in E(G)}\left(d_{u} \times d_{v}\right)$

For details see [22]. Considering the Zagreb indices, Fath-Tabar (23]) defined first and the second Zagreb polynomials as

$M_{1}(G, x)=\sum_{u v \in E(G)} x^{d_{u}+d_{v}}$

and

$M_{2}(G, x)=\sum_{u v \in E(G)} x^{d_{u} \cdot d_{v}}$

The properties of $M_{1}(G, x), M_{2}(G, x)$ polynomials for some chemical structures have been studied in the literature [24,25].

After that, in [26], the authors defined the third Zagreb index

$$
M_{3}(G)=\sum_{u v \in E(G)}\left(d_{u}-d_{v}\right)
$$

and the polynomial

$$
M_{3}(G, x)=\sum_{u v \in E(G)} x^{d_{u}-d_{v}}
$$

In the year 2016, [27] following Zagreb type polynomials were defined

$$
\begin{aligned}
& M_{4}(G, x)=\sum_{u v \in E(G)} x^{d_{u}\left(d_{u}+d_{v}\right)} \\
& M_{5}(G, x)=\sum_{u v \in E(G)} x^{d_{v}\left(d_{u}+d_{v}\right)} \\
& M_{a, b}(G, x)=\sum_{u v \in E(G)} x^{a d_{u}+b d_{v}} \\
& M_{a, b}^{\prime}(G, x)=\sum_{u v \in E(G)} x^{\left(d_{u}+a\right)\left(d_{v}+b\right)} .
\end{aligned}
$$

Ranjini et al. [28] redefines the Zagreb index, i.e, the redefined first, second and third Zagreb indices of graph $G$. These indicators appear as 


$$
\begin{aligned}
& \operatorname{Re} Z G_{1}(G)=\sum_{u v E\left(P D_{1}\right)} \frac{d_{u}+d_{v}}{d_{u} \cdot d_{v}} \\
& \operatorname{ReZG}_{2}(G)=\sum_{u v E\left(P D_{1}\right)} \frac{d_{u} \cdot d_{v}}{d_{u}+d_{v}} \\
& \operatorname{ReZG}_{3}(G)=\sum_{u v E\left(P D_{1}\right)}\left(d_{u} \cdot d_{v}\right)\left(d_{u}+d_{v}\right) .
\end{aligned}
$$

For details about topological indices and its applications we refer [29-39].

\section{Computational Results}

In this section we give our computational results.

\section{Bismuth Tri-Iodide Chain}

Theorem 1. Let $G$ be the graph of Bismuth Tri-Iodide chain $m-B i I_{3}$.Then
1. $M_{1}(G, x)=(4 m+8) x^{7}+(20 m+4) x^{8}$.
2. $M_{2}(G, x)=(4 m+8) x^{6}+(2 m+4) x^{12}$.
3. $M_{3}(G, x)=(4 m+8) x^{5}+(20 m+4) x^{4}$.
4. $M_{4}(G, x)=(4 m+8) x^{7}+(20 m+4) x^{16}$.
5. $M_{5}(G, x)=(4 m+8) x^{42}+(20 m+4) x^{48}$.
6. $M_{a, b}(G, x)=(4 m+8) x^{(a+6 b)}+(20 m+4) x^{(2 a+6 b)}$.
7. $M_{a, b}^{\prime}(G, x)=(4 m+8) x^{(1+a)(6+b)}+(20 m+4) x^{(2+a)(6+b)}$.

Proof: Let $G$ be the graph of $m-\mathrm{BiI}_{3}$ bismuth tri-iodide chain.

The edge set of $m-\mathrm{BiI}_{3}$ has following two partitions [1],

$$
\begin{aligned}
& E_{1}=E_{\{1,6\}}=\left\{e=u v \in E(G) \mid d_{u}=1, d_{v}=\right\}, \\
& E_{\{2,6\}}=\left\{e=u v \in E(G) \mid d_{u}=2, u_{v}=\right\},
\end{aligned}
$$

Now

$$
\left|E_{1}(G)\right|=4 m+8
$$$$
\left|E_{2}(G)\right|=20 m+4 \text {. }
$$ 


$$
\begin{aligned}
1 . M_{1}(G, x) & =\sum_{u v \in E(G)} x^{d_{u}+d_{v}} \\
& =\sum_{u v \in E_{1}(G)} x^{1+6}+\sum_{u v \in E_{2}(G)} x^{2+6} \\
& =\left|E_{1}(G)\right| x^{7}+\left|E_{2}(G)\right| x^{8} \\
& =(4 m+8) x^{7}+(20 m+4) x^{8} .
\end{aligned}
$$

2. $M_{2}(G, x)=\sum_{u v \in E(G)} x^{d_{u} \times d_{v}}$

$$
\begin{aligned}
& =\sum_{u v \in E_{1}(G)} x^{1 \times 6}+\sum_{u v \in E_{2}(G)} x^{2 \times 6} \\
& =\left|E_{1}(G)\right| x^{6}+\left|E_{2}(G)\right| x^{12} \\
& =(4 m+8) x^{6}+(2 m+4) x^{12} .
\end{aligned}
$$

3. $M_{3}(G, x)=\sum_{u v \in E(G)} x^{d_{v}-d_{u}}$

$$
\begin{aligned}
& =\sum_{u v \in E_{1}(G)} x^{6-1}+\sum_{u v \in E_{2}(G)} x^{6-2} \\
& =\left|E_{1}(G)\right| x^{5}+\left|E_{2}(G)\right| x^{4} \\
& =(4 m+8) x^{5}+(20 m+4) x^{4} .
\end{aligned}
$$

4. $M_{4}(G, x)=\sum_{u v \in E(G)} x^{d_{u}\left(d_{u}+d_{v}\right)}$

$$
\begin{aligned}
& =\sum_{u v \in E_{1}(G)} x^{1(1+6)}+\sum_{u v \in E_{2}(G)} x^{2(2+6)} \\
& =\left|E_{1}(G)\right| x^{7}+\left|E_{2}(G)\right| x^{16} \\
& =(4 m+8) x^{7}+(20 m+4) x^{16} .
\end{aligned}
$$


5. $M_{5}(G, x)=\sum_{u v \in E(G)} x^{d_{v}\left(d_{u}+d_{v}\right)}$

$$
\begin{aligned}
& =\sum_{u v \in E_{1}(G)} x^{6(1+6)}+\sum_{u v \in E_{2}(G)} x^{6(2+6)} \\
& =\left|E_{1}(G)\right| x^{42}+\left|E_{2}(G)\right| x^{48} \\
& =(4 m+8) x^{42}+(20 m+4) x^{48}
\end{aligned}
$$

6. $M_{a, b}(G, x)=\sum_{u v \in E(G)} x^{\left(a d_{u}+b d_{v}\right)}$

$$
\begin{aligned}
& =\sum_{u v \in E_{1}(G)} x^{(1 a+6 b)}+\sum_{u v \in E_{2}(G)} x^{(2 a+6 b)} \\
& =\left|E_{1}(G)\right| x^{(a+6 b)}+\left|E_{2}(G)\right| x^{(2 a+6 b)} \\
& =(4 m+8) x^{(a+6 b)}+(20 m+4) x^{(2 a+6 b)} .
\end{aligned}
$$

$$
\begin{aligned}
\text { 7. } M_{a, b}(G, x) & =\sum_{u v \in E(G)} x^{\left(d_{u}+a\right)\left(d_{v}+b\right)} \\
& =\sum_{u v \in E_{1}(G)} x^{(1+a)(6+b)}+\sum_{u v \in E_{2}(G)} x^{(2+a)(6+b)} \\
& =\left|E_{1}(G)\right| x^{(1+a)(6+b)}+\left|E_{2}(G)\right| x^{(2+a)(6+b)} \\
& =(4 m+8) x^{(1+a)(6+b)}+(20 m+4) x^{(2+a)(6+b)} .
\end{aligned}
$$

Theorem 2. Let $G$ be the graph of $m-B i I_{3}$. Then,

1. $\operatorname{Re} Z G_{1}(G)=\frac{54}{3} m+12$.

2. $\quad \operatorname{Re} Z G_{2}(G)=\frac{234}{7} m+\frac{90}{7}$.

3. $\operatorname{Re} Z G_{3}(G)=2088 m+720$.

\section{Proof:}


1. $\operatorname{Re} Z G_{1}(G)=\sum_{u v \in E(G)} \frac{d_{u}+d_{v}}{d_{u} \cdot d_{v}}$

$$
\begin{aligned}
& =\sum_{u v \in E_{1}(G)} \frac{1+6}{1 \cdot 6}+\sum_{u v \in E_{2}(G)} \frac{2+6}{2 \cdot 6} \\
& =\left|E_{1}(G)\right| \frac{7}{6}+\left|E_{2}(G)\right| \frac{8}{12} \\
& =\frac{7}{6}(4 m+8)+\frac{2}{3}(20 m+4) \\
& =\left(\frac{14}{3}+\frac{40}{3}\right) m+\left(\frac{28}{3}+\frac{8}{3}\right) \\
& =\frac{54}{3} m+12 .
\end{aligned}
$$

2. $\operatorname{Re} Z G_{2}(G)=\sum_{u v \in E(G)} \frac{d_{u} \cdot d_{v}}{d_{u}+d_{v}}$

$$
\begin{aligned}
& =\sum_{u v \in E_{1}(G)} \frac{1 \cdot 6}{1+6}+\sum_{u v \in E_{2}(G)} \frac{2 \cdot 6}{2+6} \\
& =\left|E_{1}(G)\right| \frac{6}{7}+\left|E_{2}(G)\right| \frac{12}{8} \\
& =\frac{6}{7}(4 m+8)+\frac{3}{2}(20 m+4) \\
& =\left(\frac{24}{7}+30\right) m+\left(\frac{48}{7}+6\right) \\
& =\frac{234}{7} m+\frac{90}{7} .
\end{aligned}
$$

3. $\operatorname{Re} Z G_{3}(G)=\sum_{u v \in E(G)}\left(d_{u} \cdot d_{v}\right)\left(d_{u}+d_{v}\right)$

$$
\begin{aligned}
& =\sum_{u v \in E_{1}(G)}(1 \cdot 6)(1+6)+\sum_{u v \in E_{2}(G)}(2 \cdot 6)(2+6) \\
& =(42)\left|E_{1}(G)\right|+(96)\left|E_{2}(G)\right| \\
& =42(4 m+8)+96(20 m+4) \\
& =168 m+336+1920 m+384 \\
& =2088 m+720 .
\end{aligned}
$$

Bismuth Tri-Iodide sheet 
Theorem 3. Let $G$ be the graph of Bismuth Tri-Iodide sheet $\mathrm{BiI}_{3}(m \times n)$.Then

1. $M_{1}(G, x)=4(m+n+1) x^{7}+4(3 m n+2 m+2 n-1) x^{8}+6 n(m-1) x^{9}$.

2. $M_{2}(G, x)=4(m+n+1) x^{6}+4(3 m n+2 m+2 n-1) x^{12}+6 n(m-1) x^{18}$.

3. $M_{3}(G, x)=4(m+n+1) x^{5}+4(3 m n+2 m+2 n-1) x^{4}+6 n(m-1) x^{3}$.

4. $M_{4}(G, x)=4(m+n+1) x^{7}+4(3 m n+2 m+2 n-1) x^{16}+6 n(m-1) x^{27}$.

5. $M_{5}(G, x)=4(m+n+1) x^{42}+4(3 m n+2 m+2 n-1) x^{48}+6 n(m-1) x^{54}$.

6. $M_{a, b}(G, x)=4(m+n+1) x^{(a+6 b)}+4(3 m n+2 m+2 n-1) x^{(2 a+6 b)}+6 n(m-1) x^{(3 a+6 b)}$.

7. $M_{a, b}^{\prime}(G, x)=4(m+n+1) x^{(1+a)(6+b)}+4(3 m n+2 m+2 n-1) x^{(2+a)(6+b)}+6 n(m-1) x^{(3+a)(6+b)}$.

Proof: Let $G$ be the graph of $\mathrm{BiI}_{3}(m \times n)$ bismuth tri-iodide sheet.

The edge set of $\mathrm{BiI}_{3}(m \times n)$ has following three partitions [1],

$$
\begin{aligned}
& E_{1}=E_{\{1,6\}}=\left\{e=u v \in E(G) \mid d_{u}=1, d_{v}=\right\}, \\
& E_{2}=E_{\{2,6\}}=\left\{e=u v \in E(G) \mid d_{u}=2, d_{v}=\right\}, \\
& E_{3}=E_{\{3,6\}}=\left\{e=u v \in E(G) \mid d_{u}=3, d_{v}=\right\},
\end{aligned}
$$

Now

$$
\begin{aligned}
& \left|E_{1}(G)\right|=4 m+4 n+4, \\
& \left|E_{2}(G)\right|=12 m n+8 m+8 n-4, \\
& \left|E_{3}(G)\right|=6 m n-6 n . \\
& \begin{aligned}
1 . M_{1}(G, x) & =\sum_{u v \in E(G)} x^{d_{u}+d_{v}} \\
& =\sum_{u v \in E_{1}(G)} x^{1+6}+\sum_{u v \in E_{2}(G)} x^{2+6}+\sum_{u v \in E_{3}(G)} x^{3+6} \\
& =\left|E_{1}(G)\right| x^{7}+\left|E_{2}(G)\right| x^{8}+\left|E_{3}(G)\right| x^{9} \\
& =(4 m+4 n+4) x^{7}+(12 m n+8 m+8 n-4) x^{8}+(6 m n-6 n) x^{9} \\
& =4(m+n+1) x^{7}+4(3 m n+2 m+2 n-1) x^{8}+6 n(m-1) x^{9} .
\end{aligned}
\end{aligned}
$$


2. $M_{2}(G, x)=\sum_{u v \in E(G)} x^{d_{u} \times d_{v}}$

$$
\begin{aligned}
& =\sum_{u v \in E_{1}(G)} x^{1 \times 6}+\sum_{u v \in E_{2}(G)} x^{2 \times 6}+\sum_{u v \in E_{3}(G)} x^{3 \times 6} \\
& =\left|E_{1}(G)\right| x^{6}+\left|E_{2}(G)\right| x^{12}+\left|E_{3}(G)\right| x^{18} \\
& =(4 m+4 n+4) x^{6}+(12 m n+8 m+8 n-4) x^{12}+(6 m n-6 n) x^{18} \\
& =4(m+n+1) x^{6}+4(3 m n+2 m+2 n-1) x^{12}+6 n(m-1) x^{18} .
\end{aligned}
$$

3. $M_{3}(G, x)=\sum_{u v \in E(G)} x^{d_{v}-d_{u}}$

$$
\begin{aligned}
& =\sum_{u v \in E_{1}(G)} x^{6-1}+\sum_{u v \in E_{2}(G)} x^{6-2}+\sum_{u v \in E_{3}(G)} x^{6-3} \\
& =\left|E_{1}(G)\right| x^{5}+\left|E_{2}(G)\right| x^{4}+\left|E_{3}(G)\right| x^{3} \\
& =(4 m+4 n+4) x^{5}+(12 m n+8 m+8 n-4) x^{4}+(6 m n-6) x^{3} \\
& =4(m+n+1) x^{5}+4(3 m n+2 m+2 n-1) x^{4}+6 n(m-1) x^{3} .
\end{aligned}
$$

4. $M_{4}(G, x)=\sum_{u v \in E(G)} x^{d_{u}\left(d_{u}+d_{v}\right)}$

$$
\begin{aligned}
& =\sum_{u v \in E_{1}(G)} x^{1(1+6)}+\sum_{u v \in E_{2}(G)} x^{2(2+6)}+\sum_{u v \in E_{3}(G)} x^{3(3+6)} \\
& =\left|E_{1}(G)\right| x^{7}+\left|E_{2}(G)\right| x^{16}+\left|E_{3}(G)\right| x^{27} \\
& =(4 m+4 n+4) x^{7}+(12 m+8 m+8 n-4) x^{16}+(6 m n-6 n) x^{27} \\
& =4(m+n+1) x^{7}+4(3 m n+2 m+2 n-1) x^{16}+6 n(m-1) x^{27}
\end{aligned}
$$

$$
\begin{aligned}
5 . M_{5}(G, x) & =\sum_{u v \in E(G)} x^{d_{v}\left(d_{u}+d_{v}\right)} \\
& =\sum_{u v \in E_{1}(G)} x^{6(1+6)}+\sum_{u v \in E_{2}(G)} x^{6(2+6)}+\sum_{u v \in E_{3}(G)} x^{6(3+6)} \\
& =\left|E_{1}(G)\right| x^{42}+\left|E_{2}(G)\right| x^{48}+\left|E_{3}(G)\right| x^{54} \\
& =(4 m+4 n+4) x^{42}+(12 m n+8 m+8 n-4) x^{48}+(6 m n-6 n) x^{54} \\
& =4(m+n+1) x^{42}+4(3 m n+2 m+2 n-1) x^{48}+6 n(m-1) x^{54} .
\end{aligned}
$$




$$
\begin{aligned}
\text { 6. } M_{a, b}(G, x) & =\sum_{u v \in E(G)} x^{\left(a d_{u}+b d_{v}\right)} \\
& =\sum_{u v \in E_{1}(G)} x^{(1 a+6 b)}+\sum_{u v \in E_{2}(G)} x^{(2 a+6 b)}+\sum_{u v \in E_{3}(G)} x^{(3 a+6 b)} \\
& =\left|E_{1}(G)\right| x^{(a+6 b)}+\left|E_{2}(G)\right| x^{(2 a+6 b)}+\left|E_{3}(G)\right| x^{(3 a+6 b)} \\
& =(4 m+4 n+4) x^{(a+6 b)}+(12 m n+8 m+8 n-4) x^{(2 a+6 b)}+(6 n m-6 n) x^{(3 a+6 b)} \\
& =4(m+n+1) x^{(a+6 b)}+4(3 m n+2 m+2 n-1) x^{(2 a+6 b)}+6 n(m-1) x^{(3 a+6 b) .}
\end{aligned}
$$

$$
\begin{aligned}
\text { 7. } M_{a, b}(G, x) & =\sum_{u v \in E(G)} x^{\left(d_{u}+a\right)\left(d_{v}+b\right)} \\
& =\sum_{u v \in E_{1}(G)} x^{(1+a)(6+b)}+\sum_{u v \in E_{2}(G)} x^{(2+a)(6+b)}+\sum_{u v \in E_{3}(G)} x^{(3+a)(6+b)} \\
& =\left|E_{1}(G)\right| x^{(1+a)(6+b)}+\left|E_{2}(G)\right| x^{(2+a)(6+b)}+\left|E_{3}(G)\right| x^{(3+a)(6+b)} \\
& =(4 m+4 n+4) x^{(1+a)(6+b)}+(12 m n+8 m+8 n-4) x^{(2+a)(6+b)}+(6 m n-6 n) x^{(3+a)(6+b)} \\
& =4(m+n+1) x^{(1+a)(6+b)}+4(3 m n+2 m+2 n-1) x^{(2+a)(6+b)}+6 n(m-1) x^{(3+a)(6+b)}
\end{aligned}
$$

Theorem 4. Let $G$ be the graph of $\mathrm{BiI}_{3}(m \times n)$. Then,

1. $\operatorname{Re} Z G_{1}(G)=11 m n+10 m+7 n+2$.

2. $\operatorname{Re} Z G_{2}(G)=30 m n+\frac{108}{7} m+\frac{24}{7} n-\frac{18}{7}$.

3. $\operatorname{Re} Z G_{3}(G)=2124 m n+936 m-36 n-216$.

\section{Proof:}


1. $\operatorname{Re} Z G_{1}(G)=\sum_{u v \in E(G)} \frac{d_{u}+d_{v}}{d_{u} \cdot d_{v}}$

$$
\begin{aligned}
& =\sum_{u v \in E_{1}(G)} \frac{1+6}{1 \cdot 6}+\sum_{u v \in E_{2}(G)} \frac{2+6}{2 \cdot 6}+\sum_{u v \in E_{3}(G)} \frac{3+6}{3 \cdot 6} \\
& =\left|E_{1}(G)\right| \frac{7}{6}+\left|E_{2}(G)\right| \frac{8}{12}+\left|E_{3}(G)\right| \frac{9}{18} \\
& =\frac{7}{6}(4 m+4 n+4)+\frac{2}{3}(12 m n+8 m+8 n-4)+\frac{1}{2}(6 m n-6 n) \\
& =(8+3) m n+\left(\frac{14}{3}+\frac{16}{3}\right) m+\left(\frac{14}{3}+\frac{16}{3}-3\right) n+\left(\frac{14}{3}-\frac{8}{3}\right) \\
& =11 m n+10 m+7 n+2 .
\end{aligned}
$$

2. $\operatorname{Re} Z G_{2}(G)=\sum_{u v \in E(G)} \frac{d_{u} \times d_{v}}{d_{u}+d_{v}}$

$$
\begin{aligned}
& =\sum_{u v \in E_{1}(G)} \frac{1 \times 6}{1+6}+\sum_{u v \in E_{2}(G)} \frac{2 \times 6}{2+6}+\sum_{u v \in E_{3}(G)} \frac{3 \times 6}{3+6} \\
& =\left|E_{1}(G)\right| \frac{6}{7}+\left|E_{2}(G)\right| \frac{12}{8}+\left|E_{3}(G)\right| \frac{18}{9} \\
& =\frac{6}{7}(4 m+4 n+4)+\frac{3}{2}(12 m n+8 m+8 n-4)+2(6 m n-6 n) \\
& =(18+12) m n+\left(\frac{24}{7}+12\right) m+\left(\frac{24}{7}\right) n+\left(\frac{24}{7}-6\right) \\
& =30 m n+\frac{108}{7} m+\frac{24}{7} n-\frac{18}{7} .
\end{aligned}
$$

3. $\operatorname{Re} Z G_{3}(G)=\sum_{u v \in E(G)}\left(d_{u} \cdot d_{v}\right)\left(d_{u}+d_{v}\right)$

$$
\begin{aligned}
& =\sum_{u v \in E_{1}(G)}(1 \cdot 6)(1+6)+\sum_{u v \in E_{2}(G)}(2 \cdot 6)(2+6)+\sum_{u v \in E_{3}(G)}(3 \cdot 6)(3+6) \\
& =(42)\left|E_{1}(G)\right|+(96)\left|E_{2}(G)\right|+(162)\left|E_{3}(G)\right| \\
& =42(4 m+4 n+4)+96(12 m n+8 m+8 n-4)+162(6 m n-6 n) \\
& =(1152+972) m n+(168+768) m+(168+768-972) n+(168-384) \\
& =2124 m n+936 m-36 n-216 .
\end{aligned}
$$

\section{Conclusions}


In the present article, we computed closed form of Zagreb polynomials for Bismuth TriIodide and then we computed redefined zagreb indices as well. Topological indices thus calculated for these Bismuth Tri-Iodide can help us to understand the physical features, chemical reactivity, and biological activities. In this point of view, a topological index can be regarded as a score function which maps each molecular structure to a real number and is used as descriptors of the molecule under testing. These results can also play a vital part in the determination of the significance of Bismuth Tri-Iodide. For example, it has been experimentally verified that the first Zagreb index is directly related with total $\pi$-electron energy.

\section{References}

1. Imran, M., Ali, M. A., Ahmad, S., Siddiqui, M. K. \& Baig, A. Q. (2018). Topological Characterization of the Symmetrical Structure of Bismuth Tri-Iodide. Symmetry 2018, 10, 201; doi:10.3390/sym10060201

2. Iodide. B. McGraw-Hill Dictionary of Scientific and Technical Terms; McGraw-Hill: New York, NY, USA, 2003.

3. Mackay, R.A.; Henderson,W. Introduction to Modern Inorganic Chemistry; CRC Press: Boca Raton, FL, USA, 2002; pp. 122-126, ISBN 0-7487-6420-8.

4. Smart, L.; Elaine, A.M. Solid State Chemistry: An Introduction; CRC Press: Boca Raton, FL, USA, 2005; p. 40, ISBN 0-7487-7516-1.

5. Watanabe, K.; Karasawa, T.; Komatsu, T.; Kaifu, Y. Optical Properties of Extrinsic Two-Dimensional Excitons in BiI3 Single Crystals. J. Phys. Soc. Jpn. 1986, 55.

6. Wyckoff, R.W.G. Crystal Structures, 2nd ed.; John Wiley \& Sons, Inc.: New York, NY, USA; London, UK; Sydney, Australia, 1964.

7. Yorikawa, H.; Muramatsu, S. Theoretical Study of Crystal and Electronic Structures of BiI3. J. Phys. Condens. Matter 2008, 20, 325-335.

8. Nason, D.; Keller, L. The Growth and Crystallography of Bismuth Tri-Iodide Crystals Grown by Vapor Transport. J. Cryst. Growth 1995, 221.

9. G. Rucker, C. Rucker, On topological indices, boiling points, and cycloalkanes. J. Chem. Inf. Comput. Sci.1999, 39, 788-802.

10. S. Klavžar, I. Gutman, A Comparison of the Schultz molecular topological index with the Wiener index. J. Chem. Inf. Comput. Sci. 1996, 36, 1001-1003. 
11. F. M. Brückler, T. Došli'c, A. Graovac, I. Gutman, On a class of distance-based molecular structure descriptors. Chem. Phys. Lett. 2011, 503, 336-338.

12. H. Deng, J. Yang, F. Xia, F. A general modeling of some vertex-degree based topological indices in benzenoid systems and phenylenes. Comp. Math. Appl.2011, 61, 3017-3023.

13. H. Zhang, F. Zhang, TheClar covering polynomial of hexagonal systems. Discret. Appl. Math. 1996, 69, 147-167.

14. I. Gutman, Some properties of the Wiener polynomials. Graph Theory Notes New York1993, 125, 13-18.

15. E. Deutsch, S. Klavzar, M-Polynomial and degree-based topological indices. Iran. J. Math. Chem. 2015, 6, 93-102.

16. M. Munir, W. Nazeer,S. Rafique, S.M. Kang, M-polynomial and related topological indices of Nanostar dendrimers. Symmetry2016, 8, 97.

17. M. Munir, W. Nazeer,S. Rafique, A. R. Nizami, S.M. Kang, M-polynomial and degree-based topological indices of titania nanotubes. Symmetry 2016, 8, 117.

18. Kwun, Y. ,Munir, M. Nazeer, W; Rafique, S.; Kang, S. M. M-polynomial and degree-based topological indices of V-phenalinic nanotubes and nanotori, Scientific Reports 7, 8756, doi:10.1038/s41598-017-08309-y2017

19. M. Munir, W. Nazeer,S. Rafique, A. R. Nizami, S.M. Kang, Some Computational Aspects of Triangular Boron Nanotubes. Symmetry 2016, 9, 6; doi: 10.3390/sym9010006.

20. M. Munir, W. Nazeer,S. Rafique, S.M. Kang, M-Polynomial and Degree-Based Topological Indices of Polyhex Nanotubes. Symmetry 2016, 8, 149.

21. Das, K. C., Xu, K., \& Nam, J. (2015). Zagreb indices of graphs. Frontiers of Mathematics in China, 10(3), 567-582.

22. I. Gutman, K. C. Das, The first Zagreb index 30 years after, MATCH-Commun. Math. Comput. Chem. 50 (2004) 83-92.

23. G. H. Fath-Tabar, Old and new Zagreb index, MATCH-Commun. Math. Comput. Chem. 65 (2011) 79-84.

24. Gutman I, Das KC. The first Zagreb index 30 years after. MATCH Commun Math Comput Chem 2004; 50: 83-92.

25. Gutman I. New bounds on zagreb indices and the zagreb co-indices. Bol Soc Paran Mat 2013; 31(1): 51-5.

26. G. H. Fath-Tabar, Zagreb Polynomial and Pi Indices of some Nano Structures, Digest Journal of Nanomaterials and Biostructures 4-1 (2009) 189-191.

27. Bindusree, A. R., Cangul, I. N., Lokesha, V., \& Cevik, A. S. (2016). Zagreb polynomials of three graph operators. Filomat, 30(7), 1979-1986. 
28. Ranjini PS, Lokesha V, Usha A. Relation between phenylene and hexagonal squeeze using harmonic index. Int J Graph Theory 2013; 1:116-21

29. Imran, M., Siddiqui, M. K., Naeem, M., \& Iqbal, M. A. (2018). On Topological Properties of Symmetric Chemical Structures. Symmetry, 10(5), 173.

30. Alaeiyan, M., Farahani, M. R., \& Jamil, M. K. (2016). Computation of the fifth geometric-arithmetic index for polycyclic aromatic hydrocarbons pahk. Applied Mathematics and Nonlinear Sciences, 1(1), 283-290.

31. Jamil, M. K., Farahani, M. R., Imran, M., \& Malik, M. A. (2016). Computing eccentric version of second zagreb index of polycyclic aromatic hydrocarbons pahkpahk. Applied Mathematics and Nonlinear Sciences, 1(1), 247-252.

32. Farahani, M. R., Jamil, M. K., \& Imran, M. (2016). Vertex PIv topological index of titania carbon nanotubes TiO2 (m, n). Appl. Math. Nonl. Sc, 1(1), 175-182.

33. Gao, W., \& Zali, M. R. (2016). Degree-based indices computation for special chemical molecular structures using edge dividing method. Applied Mathematics and Nonlinear Sciences, 1(1), 94-117.

34. Basavanagoud, B., Gao, W., Patil, S., Desai, V. R., Mirajkar, K. G., \& Balani, P. (2017). Computing First Zagreb index and F-index of New C-products of Graphs. Applied Mathematics and Nonlinear Sciences, 2(1), 285-298.

35. Lokesha, V., Deepika, T., Ranjini, P. S., \& Cangul, I. N. (2017). Operations of Nanostructures via SDD, ABC4 and GA5 indices. Applied Mathematics and Nonlinear Sciences, 2(1), 173-180.

36. Hosamani, S. M., Kulkarni, B. B., Boli, R. G., \& Gadag, V. M. (2017). QSPR analysis of certain graph theocratical matrices and their corresponding energy. Applied Mathematics and Nonlinear Sciences, 2(1), 131-150.

37. Sardar, M. S., Zafar, S., \& Zahid, Z. (2017). Computing topological indices of the line graphs of Banana tree graph and Firecracker graph. Applied Mathematics and Nonlinear Sciences, 2(1), 83-92.

38. Basavanagoud, B., Desai, V. R., \& Patil, S. (2017). ( $\beta, \alpha)$ - Connectivity Index of Graphs. Applied Mathematics and Nonlinear Sciences, 2(1), 21-30.

39. Ramane, H. S., \& Jummannaver, R. B. (2016). Note on forgotten topological index of chemical structure in drugs. Applied Mathematics and Nonlinear Sciences, 1(2), 369-374. 